\title{
Nonlinear Normal Modes of Nonconservative Systems
}

\author{
L. Renson, G. Kerschen \\ Space Structures and Systems Laboratory (S3L), Structural Dynamics Research Group \\ Department of Aerospace and Mechanical Engineering, University of Liège, Belgium
}

\begin{abstract}
Linear modal analysis is a mature tool enjoying various applications ranging from bridges to satellites. Nevertheless, modal analysis fails in the presence of nonlinear dynamical phenomena and the development of a practical nonlinear analog of modal analysis is a current research topic. Recently, numerical techniques (e.g., harmonic balance, continuation of periodic solutions) were developed for the computation of nonlinear normal modes (NNMs). Because these methods are limited to conservative systems, the present study targets the computation of NNMs for nonconservative systems. Their definition as invariant manifolds in phase space is considered. Specifically, a new finite element technique is proposed to solve the set of partial differential equations governing the manifold geometry.
\end{abstract}

Keywords: nonlinear normal modes, invariant manifold, nonconservative systems, nonlinear modal analysis.

\section{INTRODUCTION}

In many engineering applications, the efficiency of the system is critical. For structures it usually results in reducing weight, increasing flexibility, or pushing the operating range limits. As a consequence, structural dynamicists are facing new challenges in their design. One of those are nonlinearities introduced, for instance, by large displacements, nonlinear materials, damping, or gaps and joints. In this context, linear modal analysis (LMA) can still provide a useful framework to design and model the structure as long as nonlinear phenomena are negligible. In the case of essentially nonlinear systems or in the case where strong nonlinear phenomena are present, LMA fails to explain the system dynamics and results, at best, in a suboptimal design. To overcome this issue, new theoretical and numerical tools have to be developed. Among many, a specific one has drawn the attention of the structural dynamics community, namely: the Nonlinear Normal Modes (NNMs).

NNMs are mathematical tools that offers a new framework to understand nonlinear phenomena. They are a rigorous extension of the linear normal modes (LNMs) to nonlinear systems (see Section 2) and were pioneered in the 1960s by Rosenberg ${ }^{[1,2]}$. He defined an NNM as a vibration in unison of the system. Shaw and Pierre proposed a generalization of Rosenberg's definition that provides an elegant extension of the NNM concept to damped systems. Based on geometric arguments and inspired by the center manifold theory, they defined an NNM as a two-dimensional invariant manifold in phase space ${ }^{[3,4]}$.

If a large body of literature has addressed the computation of NNMs using analytical techniques (see, e.g., ${ }^{[4-8]}$ ), there have been relatively few attempts to compute NNMs using numerical methods. Most of these latter methods compute undamped NNMs, which are considered as periodic solutions of the underlying Hamiltonian system ${ }^{[9-13]}$. On the one hand, this is particularly attractive when targeting a numerical computation of the NNMs; it paves the way for the application of the NNM theory to large-scale, complex structures ${ }^{[14]}$. On the other hand, the influence of (linear and nonlinear) damping cannot be studied, which may be an important limitation in practice.

The first attempt to carry out numerical computation of damped NNMs is that of Pesheck et al. ${ }^{[15,16]}$. The manifold-governing partial differential equations (PDEs) are solved in modal space using a Galerkin projection with the NNM motion parametrized by 
amplitude and phase variables. This method eliminates a number of problems associated with the local polynomial approximation of the manifold ${ }^{[4]}$. Probably the most significant advantage is that the computation of NNMs in large-amplitude regimes can be handled. In a recent contribution, Touzé and co-workers ${ }^{[17]}$ also tackle the PDEs in modal space. They show that these PDEs can be interpreted as a transport equation, which, in turn, can be discretized using finite differences. In the study by Noreland et al. ${ }^{[18]}$, the manifold is described by partial differential algebraic equations, which are also solved by finite differences. Another interesting approach uses a Fourier-Galerkin procedure and relies on the concept of complex nonlinear modes ${ }^{[19]}$. It does not solve the governing equations of the manifold, but it is able to compute it a posteriori.

The present study introduces a new method for the computation of NNMs defined as invariant manifolds in phase space. The transformation of the manifold-governing PDEs to modal space is not necessary, which means that an NNM motion is parametrized by master displacement and velocity, as in ${ }^{[4]}$.

The approach developed in ${ }^{[16]}$ uses global shape functions to discretize the NNM PDEs, i.e. shape functions defined over the entire computational domain. The discretization ended up with a set of highly-coupled and highly-nonlinear algebraic equations to solve. As a consequence, the computational burden was dramatically increased as the number of degree-of-freedom of the mechanical system increases and a "shift in tactics" was needed ${ }^{[16]}$. A semi-discrete approach was then introduced by dividing the amplitude domain into several sub-domains in order to reduce the complexity of the shape functions in amplitude. As we are targeting the computation of NNMs for high-dimensional systems such as those met in the industry, we propose to solve the set of PDEs using the finite element (FE) method, which employs local shape functions. Thus, despite we consider physical rather than modal coordinates, our developments fits into the continuity of the work of ${ }^{[16]}$. The FE method renders the algorithm general and systematic.

The present paper is organized as follows. Section 2 briefly introduces the theoretical concepts about NNMs. Section 3 presents the details of the algorithm we developed. The FE method is presented as well as our specific treatment of boundary conditions. Section 4 validates our algorithm on a 5 degree-of-freedom (DOF) system composed of multiple nonlinearities. The validation procedure involves comparison with a continuation of periodic solution algorithm. Section 5 presents the computation of NNMs for a nonlinear system including linear and/or nonlinear damping. Finally, Section 6 concludes about the key developments and findings of this paper.

\section{REVIEW OF NORMAL MODES FOR NONLINEAR SYSTEMS}

A detailed description of NNMs and of their fundamental properties (e.g., frequency-energy dependence, bifurcations, and stability) is given in ${ }^{[7,20]}$ and is beyond the scope of this paper. For completeness, the two main definitions of an NNM are briefly reviewed in this section.

The free response of discrete mechanical systems with $N$ degrees of freedom (DOFs) is considered, assuming that continuous systems (e.g., beams, shells, or plates) have been spatially discretized using the FE method. The equations of motion are

$$
\mathbf{M} \ddot{\mathbf{x}}(t)+\mathbf{G}_{\mathrm{gyr}} \dot{\mathbf{x}}+\mathbf{C} \dot{\mathbf{x}}(t)+\mathbf{K} \mathbf{x}(t)+\mathbf{f}_{\mathrm{nl}}\{\mathbf{x}(t), \dot{\mathbf{x}}(t)\}=0
$$

where $\mathbf{M}, \mathbf{G}_{\text {gyr }}, \mathbf{C}$, and $\mathbf{K}$ are the mass, gyroscopic coupling, damping, and stiffness matrices, respectively; $\mathbf{x}, \dot{\mathbf{x}}$, and $\ddot{\mathbf{x}}$ are the displacement, velocity, and acceleration vectors, respectively; $\mathbf{f}_{n \mathrm{n}}$ is the nonlinear restoring force vector.

Targeting a straightforward nonlinear extension of the concept of LNMs, Rosenberg defined an NNM motion as a synchronous periodic oscillation. This definition requires that all material points of the system reach their extreme values and pass through zero simultaneously and allows all displacements to be expressed in terms of a single reference displacement. At first glance, Rosenberg's definition may appear restrictive in two cases:

1. In the presence of internal resonances, an NNM motion is no longer synchronous, but it is still periodic. This is why an extended definition was considered in ${ }^{[10,20]}$; an NNM motion was defined as a (non-necessarily synchronous) periodic motion of the undamped mechanical system.

2. The definition cannot be easily extended to nonconservative systems. However, as shown in ${ }^{[20]}$, the damped dynamics can be interpreted based on the topological structure of the NNMs of the underlying conservative system, provided that damping has a purely parasitic effect. 
For illustration, the system depicted in Figure 1 and governed by the equations

$$
\begin{aligned}
\ddot{x}_{1}+\left(2 x_{1}-x_{2}\right)+0.5 x_{1}^{3} & =0, \\
\ddot{x}_{2}+\left(2 x_{2}-x_{1}\right) & =0,
\end{aligned}
$$

is considered. The NNMs corresponding to in-phase and out-of-phase motions are represented in the frequency-energy plot (FEP) of Figure 2. An NNM motion is represented by a point in the FEP, which is drawn at a frequency corresponding to the minimal period of the periodic motion and at an energy equal to the conserved total energy during the motion. A branch, represented by a solid line, is a family of NNM motions possessing the same qualitative features (e.g., in-phase NNM motion).

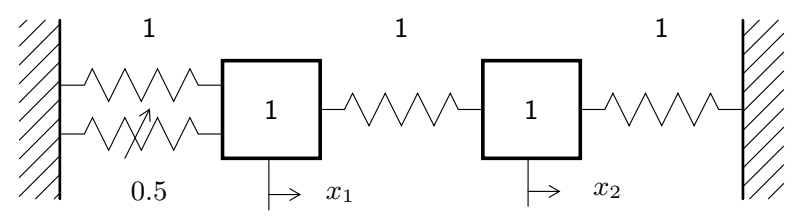

Figure 1: Schematic representation of the 2DOF system example.

To provide a rigorous extension of the NNM concept to damped systems, Shaw and Pierre defined an NNM as a two-dimensional invariant manifold in phase space ${ }^{[3]}$. Such a manifold is invariant under the flow (i.e., orbits that start out in the manifold remain in it for all time), which generalizes the invariance property of LNMs to nonlinear systems. In order to parametrize the manifold, a single pair of state variables (i.e., both the displacement and the velocity) are chosen as master coordinates, the remaining variables being functionally related to the chosen pair. Therefore, the system behaves like a nonlinear single-DOF system on the manifold.

Geometrically, LNMs are represented by planes in phase space, and NNMs are two-dimensional surfaces that are tangent to them at the equilibrium point. The bending and contortion of a NNM surface is purely due to nonlinear effects. The invariant manifolds corresponding to in-phase and out-of-phase NNM motions of system (2) are given in Figure 3.

\subsection{Manifold-governing partial differential equations}

To derive the equations governing the geometry of the manifold, Equations (1) are recast into state-space form:

$$
\begin{aligned}
\dot{x}_{i} & =y_{i}, \\
\dot{y}_{i} & =f_{i}(\mathbf{x}, \mathbf{y}), \quad i=1, \ldots, N .
\end{aligned}
$$

The formulation and notations presented here closely follow those used in reference ${ }^{[4]}$. The $f_{i}(\mathbf{x}, \mathbf{y})$ accounts for all the forcing terms presented in the equations of motion: linear stiffness terms, linear gyroscopic coupling terms, linear damping terms, and nonlinear stiffness and damping terms.

During an NNM motion, there is a functional dependence between all degrees of freedom, and the motion can be parametrized by a single displacement-velocity pair. The selection of the master coordinates $(u, v)=\left(x_{k}, y_{k}\right)$, i.e., the nonlinear modal coordinates, is arbitrary. The $2 N-2$ constraint equations governing the slave coordinates are:

$$
\begin{aligned}
x_{i} & =X_{i}(u, v), \\
y_{i} & =Y_{i}(u, v), \quad i=1, \ldots, N ; i \neq k .
\end{aligned}
$$

To obtain a set of equations governing the manifold geometry, i.e., the $X_{i}$ 's and $Y_{i}$ 's, the time dependence in the equations is eliminated. Taking the time derivative of the constraint equations (4) and using the chain rule for differentiation yields:

$$
\begin{aligned}
\dot{x}_{i} & =\frac{\partial X_{i}}{\partial u} \dot{u}+\frac{\partial X_{i}}{\partial v} \dot{v}, \\
\dot{y}_{i} & =\frac{\partial Y_{i}}{\partial u} \dot{u}+\frac{\partial Y_{i}}{\partial v} \dot{v}, \quad i=1, \ldots, N ; i \neq k .
\end{aligned}
$$

Plugging Equations (3) and (4) into Equation (5) leads to a set of $2 \mathrm{~N}-2$ partial differential equations (PDEs) that can be solved for 


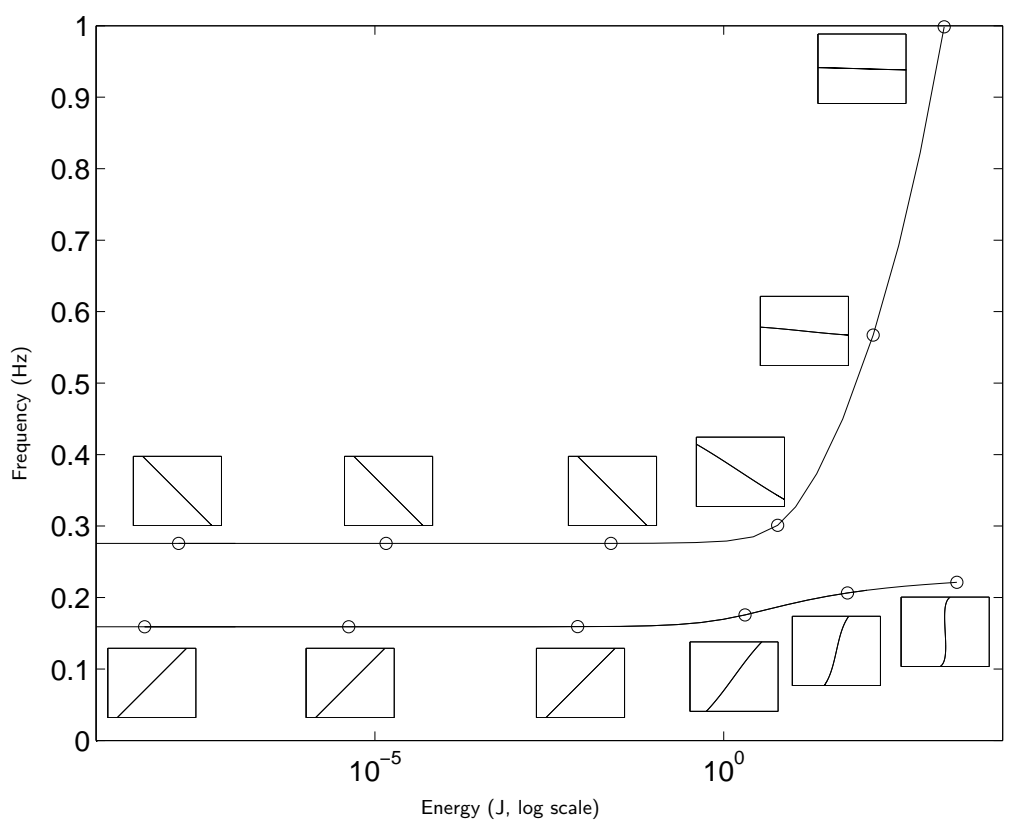

Figure 2: Frequency-energy plot of system (2). NNM motions depicted in the configuration space are inset.

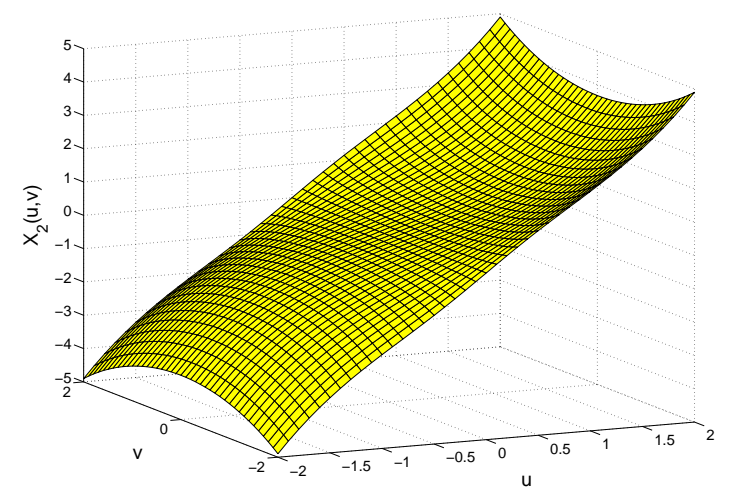

(a)

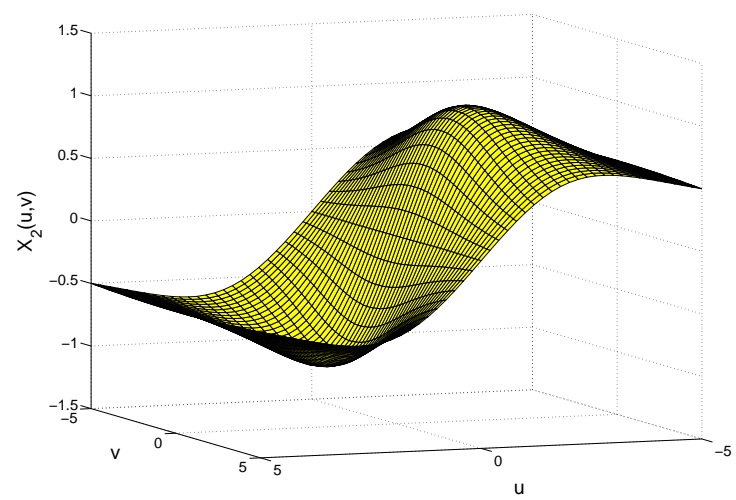

(b)

Figure 3: Two-dimensional invariant manifolds of system (2) computed with an algorithm for the continuation of periodic solutions. The different periodic solutions computed form orbits in the phase space that paves the manifold. (a) In-phase NNM; and (b) out-of-phase NNM.

the $X_{i}$ 's and $Y_{i}^{\prime}$ 's:

$$
\begin{aligned}
Y_{i}(u, v)= & \frac{\partial X_{i}(u, v)}{\partial u} v+\frac{\partial X_{i}(u, v)}{\partial v} f_{k}(u, \mathbf{X}(u, v), v, \mathbf{Y}(u, v)), \\
f_{i}(u, \mathbf{X}(u, v), v, \mathbf{Y}(u, v))= & \frac{\partial Y_{i}(u, v)}{\partial u} v+\frac{\partial Y_{i}(u, v)}{\partial v} f_{k}(u, \mathbf{X}(u, v), v, \mathbf{Y}(u, v)), \\
& i=1, \ldots, N ; i \neq k
\end{aligned}
$$


where $\mathbf{X}=\left\{X_{j}: j=1, \ldots, N ; j \neq k\right\}$ and $\mathbf{Y}=\left\{Y_{j}: j=1, \ldots, N ; j \neq k\right\}$. Equations for $i=k$ are trivially satisfied.

Once the manifold-governing PDEs are solved, constraint equations (4) represent the geometrical description of the NNM. Around the equilibrium point, Equations (6) admit $N$ solutions, i.e., one for each mode. The nonlinear modal dynamics is then generated by substituting the $X_{i}$ 's and $Y_{i}^{\prime}$ 's in the pair of equations of motion governing the master coordinates $x_{k}$ and $y_{k}$. This results in a single-DOF nonlinear motion:

$$
\begin{aligned}
\dot{u} & =v \\
\dot{v} & =f_{k}(u, \mathbf{X}(u, v), v, \mathbf{Y}(u, v)), \quad i=1, \ldots, N ; i \neq k
\end{aligned}
$$

Here, we mention that the approach followed to derive NNMs PDEs relies on the assumption of a two-dimensional description of the dynamics. For some regime of motion, often away from the equilibrium point, this assumption is not true. It results in a failure of the PDE description and in an inability to compute the NNMs in these regions. This limitation influences the selection of the master coordinates that are, in fact, not arbitrary. The observation of reference solutions show that the maximum computational domain is (strongly) influenced by this choice. Thus, taking the appropriate master coordinates ensure results with a large validity range. Unfortunately, there is not yet good practices to identify the most appropriate set of coordinates.

\section{FINITE ELEMENT COMPUTATION OF NONLINEAR NORMAL MODES}

To fit into a formulation more convenient to the FEM, Equations (6) are recast into Equations (8) where $\nabla$ is the gradient vector, and $\partial \Omega$ the domain boundary. This boundary comprises an inflow boundary $\partial \Omega^{-}=\{\mathbf{u} \in \partial \Omega: \mathbf{V}(\mathbf{u}) . \mathbf{n}(\mathbf{u})<0\}$ and an outflow boundary $\partial \Omega^{+}=\{\mathbf{u} \in \partial \Omega: \mathbf{V}(\mathbf{u}) \cdot \mathbf{n}(\mathbf{u})>0\}$. The $2 \mathrm{~N}-2$ equations are quasilinear first order partial differential equations that are similar to flow equations encountered in fluid dynamics. This interpretation of the PDEs is identical to the interpretation made by Touzé and its co-workers ${ }^{[17]}$ who interpreted the PDEs as a transport problem. The vector $\mathbf{V}$ can therefore be interpreted as the velocity vector of the master coordinates flow. Transforming the PDEs in Equations (8) into (9), a new matrix $\mathbf{A}$ is bring out where $\mathbf{I}$ is the identity matrix and $\mathbf{F}^{T}=\left\{f_{j}: j=1, \ldots, N ; j \neq k\right\}$. It is a diagonal matrix which has a single real eigenvalue $\lambda=f_{k} / v$ of multiplicity $2 \mathrm{~N}-2$. The eigenvalues of $\mathbf{A}$ can be interpreted as the flow speed. Here, the velocity amplitude and direction is identical for every unknown field $X_{i}$ or $Y_{i}$. The flow direction is called the characteristic direction and illustrates the propagation of the information into the domain ${ }^{[21-23]}$.

$$
\begin{aligned}
& \left\{\begin{array}{c}
\mathbf{V} \cdot \nabla X_{i}-Y_{i}=0 \\
\mathbf{V} \cdot \nabla Y_{i}-f_{i}=0 \\
\left.X_{i}\right|_{\partial \Omega^{-}}=X_{i}^{-} \\
\left.Y_{i}\right|_{\partial \Omega^{-}}=Y_{i}^{-}
\end{array}\right. \\
& i=1, \ldots, N ; i \neq k, \quad \mathbf{V}^{T}=\left\{f_{k} \quad v\right\}
\end{aligned}
$$

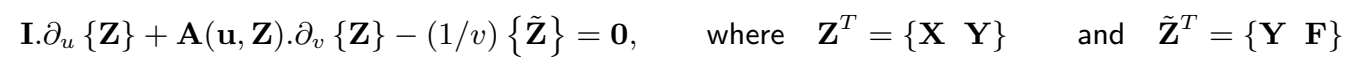

Figure 4 (a) shows the flow of a 2DOF nonlinear conservative system. In the domain $\Omega$, characteristic directions form curves called characteristic curves (for short, characteristics). In the present case, the conservation of the energy induces closed characteristics. The information travels through out the domain along these curves. We conclude in a clear distinction between the dynamics of different energies. Indeed, the "information" related to a given energy is limited to the corresponding characteristic. This illustrates there are no mixing between different energy dynamics; high and low energy dynamics do not influence each other. In the non-conservative case, characteristics spiral toward the equilibrium point of the system (here, the origin) (see Figure 4 (b)).

Solving hyperbolic PDEs requires boundary conditions at inflow $\left(\partial \Omega^{-}\right)$, i.e. where the velocity vector $\mathbf{V}$ points inward the domain. In our first order problem, it requires to set the unknown field values $\left(X_{i}^{-}, Y_{i}^{-}\right)$at this boundary. However, the domain boundary is away from the equilibrium point and corresponds to a region of high-energy where no theoretical information about the manifold is available. A first classical strategy would use an extrapolation techniques to estimate the unknown values at the boundary using the solution computed inside the domain. According to the above interpretation in terms of characteristics, this will involve a mixing between different energy dynamics. Extrapolation was therefore neglected. Nevertheless, we note that a domain with an iso-energy curve for boundary remains everywhere tangent to the flow and boundary conditions become obsolete. Assuming two different iso-energy curves, they form the inner and outer boundaries of an annular domain. Since a clear partition of the dynamics exists, one can solve the PDEs in this region disregarding the solution computed outside it. This strategy is key to our algorithm. It avoids to set disruptive boundary conditions and reduces the computational burden. This is particularly interesting as the number of degree-of-freedom of the mechanical system becomes large. 


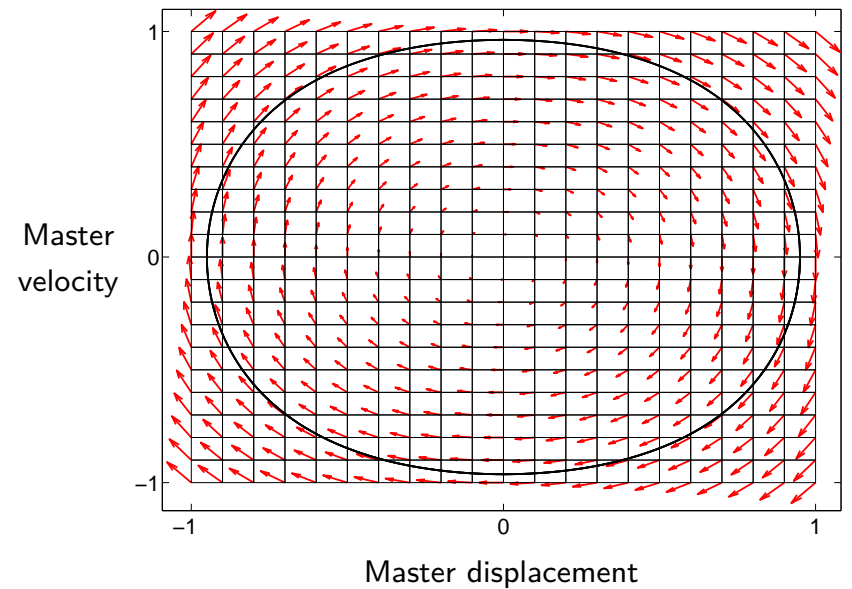

(a)

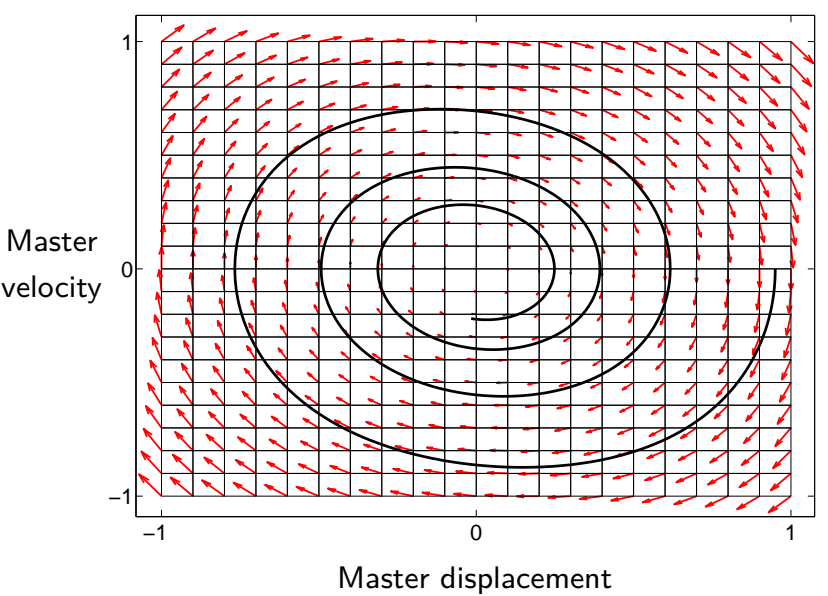

(b)

Figure 4: PDEs are interpreted as flow equations where $\mathrm{V}$ describes the velocity field $(\rightarrow)$. (a) For a conservative system, the flow is tangent to iso-energy curves. (b) For a nonconservative system, the flow spirals to the equilibrium point.

For noncoservative systems, there is no partition of the dynamics and the flow points inward the domain boundary. However, the methodology suggested remains applicable. Indeed, let's take an annular domain whose inner and outer boundaries correspond to iso-energy curves. Generally speaking, the flow points in and out at the outer and inner boundaries; respectively. However, from a pure mathematical point of view, characteristics only specify directions. It means that the flow can be "inverted" and spiral in and out at the inner and outer boundary; respectively. According to this observation, we can solve an "inverse" problem where boundary conditions have to be specified at the inner boundary. A recursive strategy can then be applied to progressively solve the PDEs using previously computed solution as boundary conditions for the next domain.

The propose strategy suffers from a single limitation that is: iso-energy curves are only known at the solution. To circumvent this issue, a first guess of the iso-energy curve is computed using the previous domain boundary and solution. Then the shape of the new computational domain is iteratively modified as the new solution is computed. At the solution, the domain boundary fits the actual iso-energy.

\subsection{Resolution strategy overview}

The resolution strategy is illustrated in Figure 5. The computation starts in a small domain around the origin where the system behaves almost linearly. The domain boundary is established for a given initial energy and computed using LNM. The LNM selected corresponds to the targeted NNM. The LNM is also considered as a first guess for the solution. To compute the actual solution, the domain boundary is corrected in order to fit the iso-energy corresponding to the current estimation of the solution. The correction is performed thanks to a mesh moving technique. It is applied a user-defined number of times and until some criteria are fulfilled (see Section 3.4). Domain corrections precedes each correction of the PDE solution in order to satisfy the boundary conditions. Once the solution is computed within a given accuracy tol, a new annular domain can be predicted. The prediction of the next iso-energy is performed using the previously computed solution. The new curve is considered as the new outer boundary whereas the old outer boundary becomes the inner one. Both boundaries define the new computational domain. The solution on this new annular region can be computed following a similar sequence of domain-correction and solution-correction. The NNM computation stops when the number of domain to compute or a maximum energy has been reached. Post-processing is performed in order to merge the different annular domains computed.

\subsection{Streamline Upwinded Petrov-Galerkin}

In order to solve hyperbolic PDEs using the FEM, specific numerical techniques are employed. Indeed, classical Galerkin finite element formulations using similar shape and test function spaces have demonstrated poor results, for instance, in the context of 


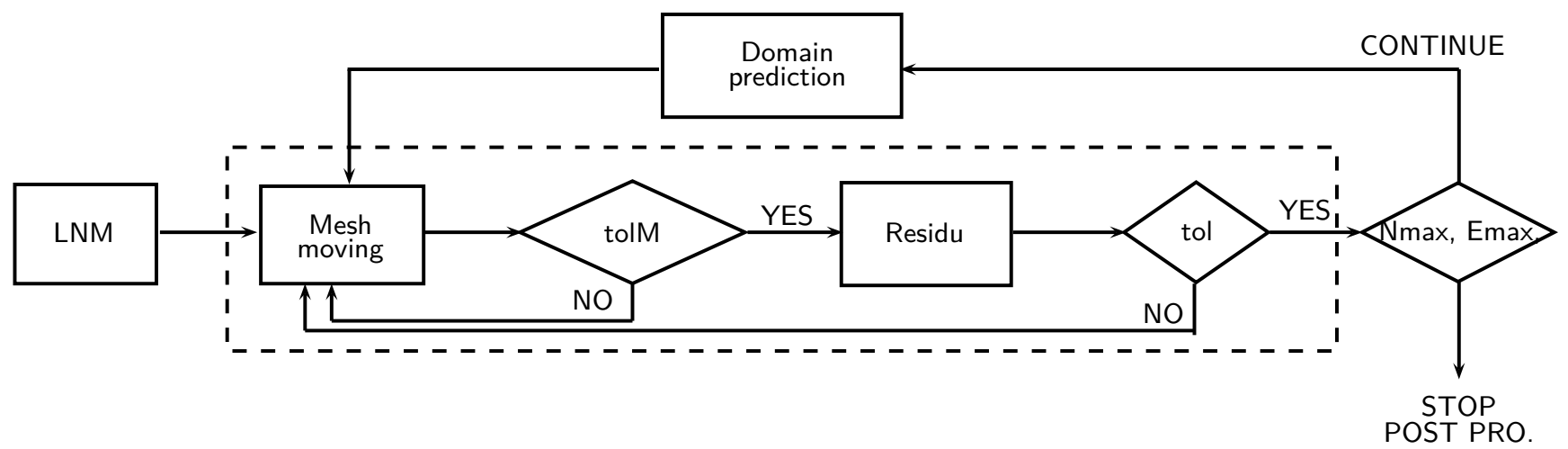

Figure 5: Schematics of the algorithm resolution strategy.

fluid dynamics ${ }^{[23,24]}$. In the Finite Difference (FD) framework, off-centered schemes in the upwind direction are used in order to account for the "transport" phenomenon. In a similar way, several finite element formulations deal with this transport phenomenon using alternative test functions. The SUPG method is used herein and consists in over-weighting the shape functions that are upstream. This method falls within the general category of Petrov-Galerkin formulations where test and shape functions are taken in different spaces. The approach is rather standard and out of the scope of our paper. We briefly present the major points of our formulation and the interested reader can refer to ${ }^{[23-25]}$ for practical details about SUPG.

To derive our finite element formulation, a weighted residual approach is applied to Equations (8). The formulation is presented in Equations (10) where $i=1, \ldots, N$ with $i \neq k$ and where shape functions are considered as simple first order Lagrange shape functions: $N^{b} \in \mathbb{P}^{1}$. The test functions are $\tilde{N}^{b}=N^{b}+\tau \mathbf{V} \cdot \nabla \mathbf{N}^{\mathbf{b}}$. As already mentioned, test functions differ from classical Galerkin test functions via an upstream overweighting $\left(\tau \mathbf{V} \cdot \nabla \mathbf{N}^{\mathbf{b}}\right)$. As a consequence, discontinuous test functions are employed ${ }^{[25]}$. Such test functions are illustrated in Figure 6 and naturally account for the upstream origin of the information transported throughout the domain.

$$
\begin{aligned}
\iint_{\Omega}\left[\mathbf{V}(u, v, \mathbf{X}, \mathbf{Y}) \cdot \nabla X_{i}(u, v)-Y_{i}(u, v)\right] \delta \tilde{Y}_{i} d u d v & =0 \\
\iint_{\Omega}\left[\mathbf{V}(u, v, \mathbf{X}, \mathbf{Y}) \cdot \nabla Y_{i}(u, v)-f_{i}(u, v, \mathbf{X}, \mathbf{Y})\right] \delta \tilde{X}_{i} d u d v & =0
\end{aligned}
$$

The parameter $\tau$ is a problem dependent parameter. In fluid dynamics, it is often determined using Equation (11) which is a function of the Peclet element number $\mathrm{Pe}^{h}$, i.e. the ratio between diffusion and advection over the element, and $h$ is the characteristic size of the elements. In the present case, our problem does not involve any diffusion and the limit for $\operatorname{Pe}^{h} \rightarrow \infty$ is $\tau=\frac{h}{2\|V\|_{2}}$. The estimation of the parameter $\tau$ is an important topic which still gives rise to recent publications ${ }^{[26]}$. Indeed, various other possible definitions for $\tau$ are presented in the literature ${ }^{[27-29]}$. According to this literature, the value of $\tau$ has a strong influence on the quality of the results. According to preliminary tests, the accuracy of the algorithm for NNM computation is not critically influenced by $\tau$. However, the speed of convergence is. Further investigations on this parameter have to be performed.

$$
\tau=\frac{h}{2\|V\|_{2}}\left(\operatorname{coth}\left(\mathrm{Pe}^{h}\right)-\frac{1}{\mathrm{Pe}^{h}}\right)
$$

After the FE discretization, the set of PDEs is transformed to a set of coupled nonlinear algebraic equations. These equations are solved using a Newton-Raphson procedure where the Jacobian matrix is provided analytically. An important feature of our problem is that equations to solve remains nonlinear despite the mechanical system considered is linear.

In practice, master displacement and velocity frequently have different scales as it is commonly encountered in mechanical systems. This difference results in a domain with a poor aspect-ratio which, in turn, requires an excessive number of mesh elements. Two different scalings are applied to overcome this issue. The first one scales the time in order to retrieve similar displacement and velocity scales. The second scaling modifies both displacements and velocities in order to make them of order unity. Since such scalings are standard, they are not further discussed. 


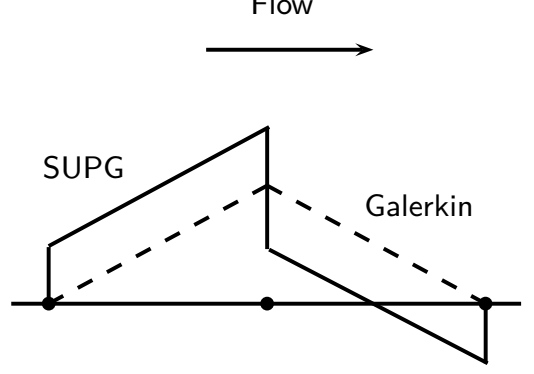

Figure 6: Comparison between test functions in a classical Galerkin formulation (- - ) and the discontinuous test functions in the SUPG formulation (-).

\subsection{Domain prediction}

The prediction of the domain $d+1$ is performed using an approach similar to continuation. We first assume that the solution computed on the domain $d$ is accurate. The outer boundary is therefore an iso-energy curve of energy $E^{d}$. This outer boundary becomes the inner boundary of the next domain $d+1$. To compute the new outer boundary, we apply an energy increment $\Delta E^{d}$ and look for an estimation of the iso-energy curve at $E^{d+1}=E^{d}+\Delta E^{d}$. Linearizing the energy around each point $i$ of the boundary $d$, we obtain Equation (12). Solving Equation (13) transforms the energy increment in terms of displacements $\left(\Delta u_{i}, \Delta v_{i}\right)$ to apply to the boundary nodes. Figure 7 (a) illustrates the result of this procedure.

$$
\begin{array}{r}
E^{d+1}=E^{d}+\left.\frac{d E}{d u}\right|^{d, i} \Delta u_{i}+\left.\frac{d E}{d v}\right|^{d, i} \Delta v_{i}, \quad \forall i \\
\Rightarrow \Delta E^{d}=\left.\frac{d E}{d u}\right|^{d, i} \Delta u_{i}+\left.\frac{d E}{d v}\right|^{d, i} \Delta v_{i}, \quad \forall i .
\end{array}
$$

Solving for $(\Delta u, \Delta v)$ requires the use of the "Moore-Penrose" pseudo-inverse since the system is under determined. The sensitivity of the energy with respect to $u$ and $v$ is presented in Equations $(14-15)$ where the constraint relations between slave and master coordinates are considered. The sensitivity of the energy with respect to master or slave coordinates has a clear link with the equations of motion (1) and is provided analytically. The sensitivity of the constraint relations (i.e. $\partial_{u} X, \ldots$ ) are provided by the FEM. Note that linear and nonlinear nonconservative terms are disregarded in the equations. Finally, an estimation of the unknown field $j$ is provided thanks to the local tangent-plane at each node $i$ (Equations (16)). The result is illustrated in Figure 7 (b).

$$
\begin{gathered}
E_{i}=E_{i}(u, v)=E_{i}(u, v, \mathbf{X}(u, v), \mathbf{Y}(u, v))=\frac{1}{2}\left(\mathbf{q}^{T} \mathbf{K} \mathbf{q}+\dot{\mathbf{q}}^{T} \mathbf{M} \dot{\mathbf{q}}+\dot{\mathbf{q}}^{T} \mathbf{G}_{\mathrm{gyr}} \dot{\mathbf{q}}+E_{\mathrm{NL}}\right) . \\
\frac{d E_{i}}{d u}=\left.\frac{\partial E}{\partial u}\right|_{i}+\left.\left.\frac{\partial E}{\partial \mathbf{X}}\right|_{i} \frac{\partial \mathbf{X}}{\partial u}\right|_{i}+\left.\left.\frac{\partial E}{\partial \mathbf{Y}}\right|_{i} \frac{\partial \mathbf{Y}}{\partial u}\right|_{i}, \\
\frac{d E_{i}}{d v}=\left.\frac{\partial E}{\partial v}\right|_{i}+\left.\left.\frac{\partial E}{\partial \mathbf{X}}\right|_{i} \frac{\partial \mathbf{X}}{\partial v}\right|_{i}+\left.\left.\frac{\partial E}{\partial \mathbf{Y}}\right|_{i} \frac{\partial \mathbf{Y}}{\partial v}\right|_{i} \\
X_{j}^{d+1}\left(u_{i}^{d+1}, v_{i}^{d+1}\right)=X_{j}^{i}\left(u_{i}^{d}, v_{i}^{d}\right)+\left.\frac{\partial X_{j}}{\partial u}\right|_{i} \Delta u_{i}+\left.\frac{\partial X_{j}}{\partial v}\right|_{i} \Delta v_{i} \\
Y_{j}^{d+1}\left(u_{i}^{d+1}, v_{i}^{d+1}\right)=Y_{j}^{i}\left(u_{i}^{d}, v_{i}^{d}\right)+\left.\frac{\partial Y_{j}}{\partial u}\right|_{i} \Delta u_{i}+\left.\frac{\partial Y_{j}}{\partial v}\right|_{i} \Delta v_{i}, \\
\forall i, \quad j=1, \ldots, N ; j \neq k .
\end{gathered}
$$

\subsection{Domain correction using a mesh moving technique}

As introduced, the shape of the computational domain is chosen in order to avoid to impose boundary conditions. Such a domain needs to be everywhere tangent to the flow $\mathbf{V}$. In the particular case of a conservative system, the flow is everywhere tangent to iso-energy curves. An iso-energetic domain boundary would therefore meet our requirement. Unfortunately, such curves are known at 


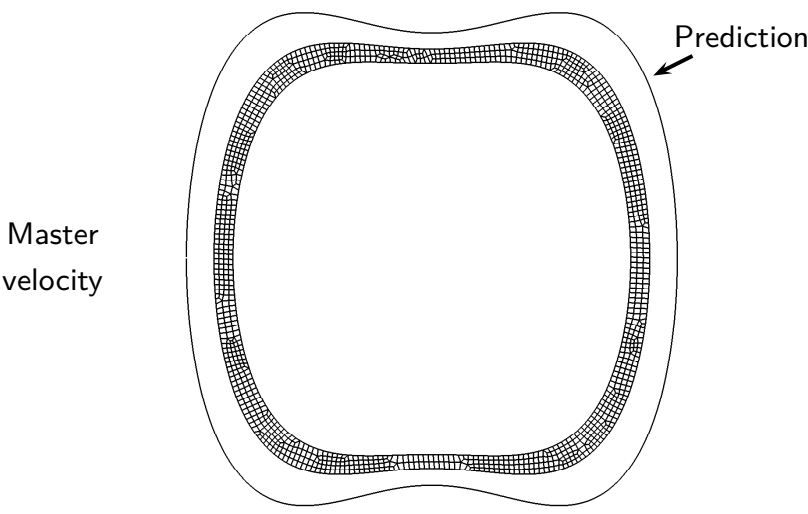

Master displacement

(a)

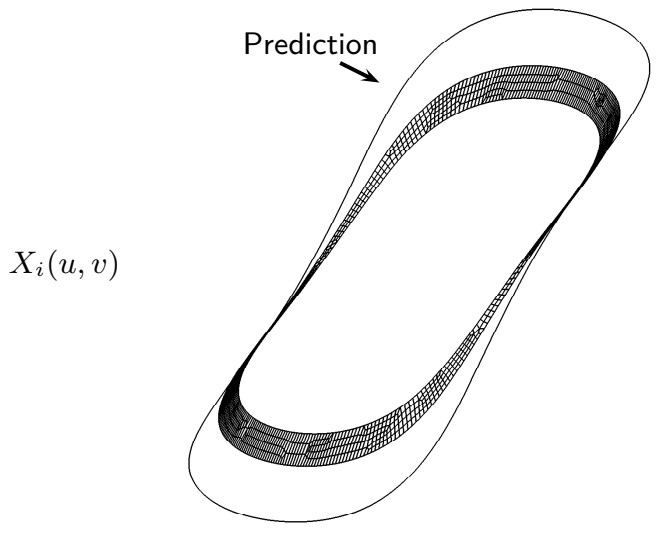

(b)

Figure 7: From the solution computed for the annular domain, we predict the next domain boundary (a) and the values of the different unknown fields (b).

the solution and only a prediction of it can be performed. In order to satisfy the need of boundary conditions to solve the hyperbolic problem, the domain is modified at each iteration such that it is an iso-energy curve for the current estimation of the solution. At each iteration, BCs are thus satisfied and the hyperbolic problem is well posed.

In order to modify the domain's shape so that it fits closely an iso-energy curve, the mesh is considered as a pseudo-elastic medium and a linear elasticity problem is solved. The inner boundary is considered as clamped (displacements are set to zero) while the outer boundary is moved in order to equal a reference energy. For simplicity reasons, the reference energy is considered to be the energy of the node with $u_{\max }=\max (u)$. This reference energy evolves with the computation of the solution. The formula used to compute the displacements imposed to the boundary nodes are similar to formula used for domain prediction (Equations (12 - 15)). The only difference is the energy increment $\Delta E$ that is now determined to equal the reference energy.

Mesh moving techniques have the advantage to properly deform the domain so that elements remain well-shaped and that (almost) no remeshing operations are needed. Without going into the details of mesh moving techniques, the equations governing the displacements are:

$$
\begin{gathered}
\nabla \cdot \sigma=\mathbf{0}, \quad \sigma=\mathbf{D}: \epsilon, \quad \epsilon=\frac{1}{2}\left(\nabla \mathbf{q}+\nabla \mathbf{q}^{\top}\right), \\
\mathbf{D}_{i j k l}=\frac{E \nu}{(1+\nu)(1-2 \nu)} \delta_{i j} \delta_{k l}+\frac{E}{1+\nu}\left(\frac{1}{2} \delta_{i k} \delta_{j l}+\frac{1}{2} \delta_{i l} \delta_{j k}\right),
\end{gathered}
$$

where $\nabla$.( ) denotes the divergence operator, $\sigma$ the Cauchy stress tensor, $\mathbf{D}$ the Hooke tensor, $\epsilon$ the strain tensor, $E$ Young's modulus, $\nu$ Poisson's ratio, and $\delta_{i j}$ the Kronecker delta. After discretization using the finite element method, a linear algebraic problem is obtained (Equation (18)) where $\mathbf{K}^{\text {elast }}$ denotes the stiffness matrix of the discretized domain considered as a pseudo-elastic medium. This matrix should not be confused with the stiffness matrix $\mathbf{K}$ of the mechanical system.

$$
\mathbf{K}^{\text {elast }} \mathbf{q}=\mathbf{0}
$$

The displacements $\mathbf{q}$ are split into three different sets, namely: clamped nodes whose displacements equal zero $\left(\mathbf{q}_{0}=\mathbf{0}\right)$, outer nodes whose displacements are non-zero and follow the energy corrections $\left(\mathbf{q}_{d}\right)$, and finally internal nodes whose displacements are unknown $\left(\mathbf{q}_{i}\right)$. This partition provides a straight forward expression for the unknown displacements $\mathbf{q}_{i}$ (Equation (19)). Additional information about mesh moving techniques can be found in ${ }^{[30,31]}$.

$$
\left[\begin{array}{lll}
\mathbf{K}_{i i} & \mathbf{K}_{i d} & \mathbf{K}_{i 0} \\
\mathbf{K}_{d i} & \mathbf{K}_{d d} & \mathbf{K}_{d i} \\
\mathbf{K}_{0 i} & \mathbf{K}_{0 d} & \mathbf{K}_{00}
\end{array}\right]^{\text {elast }}\left[\begin{array}{c}
\mathbf{q}_{i} \\
\mathbf{q}_{d} \\
\mathbf{q}_{0}
\end{array}\right]=\mathbf{0} \Rightarrow \mathbf{q}_{i}=-\mathbf{K}_{i i}^{-1} \mathbf{K}_{i d} \mathbf{q}_{d}
$$


Mesh modification according to iso-energy curves is critical and can lead to divergence if not performed. Indeed, the correction should be applied enough times in order to have a domain which is consistent with the current estimation of the solution. Without proper boundary conditions, the problem to solve is not well posed and the PDE resolution will fail. The mesh-moving algorithm is applied, at least, a user-defined number of times. It is stopped if the mean energy of all boundary points is sufficiently close to the reference energy. An additional criteria can be set on the standard deviation of the energy to ensure that boundary points have indeed an energy close to the reference one.

\section{ALGORITHM DEMONSTRATION ON CONSERVATIVE EXAMPLES}

In this section, the NNMs of a five DOFs conservative system are discussed and used to assess our resolution method. The system considered includes a cubic spring between the second and third masses. It also includes a quadratic and quintic spring between the third and fifth masses. The system is illustrated in Figure 8 with its numerical parameters.

For conservative systems, different reference algorithms to compute NNMs exist ${ }^{[11,13,32]}$. For instance, the "exact" manifolds can be computed using the technique developed in ${ }^{[13]}$, which combines shooting and pseudo-arclength continuation. For each NNM, the graphical depiction in phase space of the periodic orbits at different energy levels provides a reference solution to which we can compare the solution computed using the invariant manifold approach.

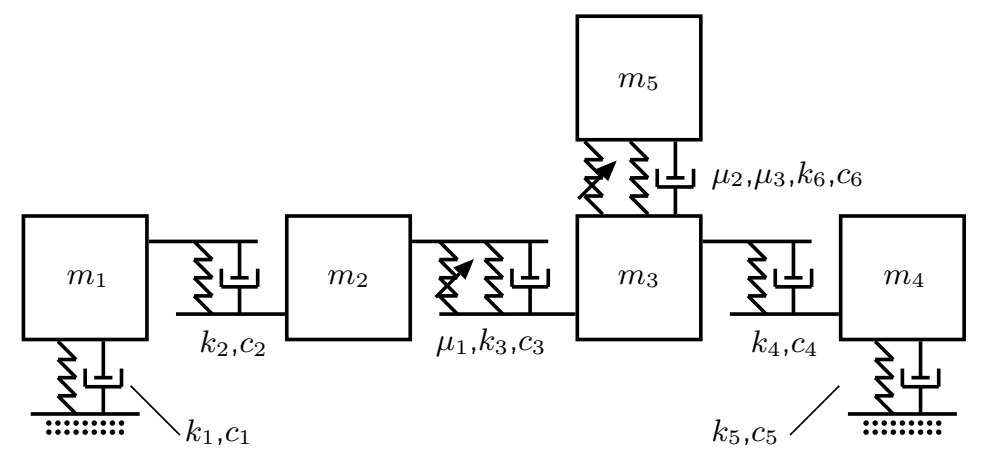

Figure 8: Schematic view of the 5 DOF system. A cubic nonlinearity with coefficient $\mu_{1}$ is introduced between the second and the third masses. A quadratic and quintic nonlinearity is introduced between the third and the fifth masses $\left(\mu_{2}, \mu_{3}\right)$. $m_{1-6}=1[\mathrm{Kg}], k_{1-5}=50 e 3[\mathrm{~N} / \mathrm{m}], k_{6}=35 e 3[\mathrm{~N} / \mathrm{m}], c_{1-6}=0[\mathrm{Ns} / \mathrm{m}], \mu_{1}=500 e 6\left[\mathrm{~N} / \mathrm{m}^{3}\right], \mu_{2}=-500 e 3\left[\mathrm{~N} / \mathrm{m}^{2}\right]$, $\mu_{3}=10 e 9\left[N / m^{5}\right]$.

Figure 9 presents the second NNM of the 5DOF system for the slave displacement and velocity of the third mass $\left(X_{3}\right.$ and $\left.Y_{3}\right)$. The results coming from the invariant manifold approach are depicted in green whereas the reference solutions are in yellow. A very good agreement between computed and reference surfaces is observed (green and yellow are "intimately mixed").

A more quantitative comparison of the results is now carried out. The initial conditions in the modal space $(u, v)$ of the second mode are transformed back to physical space (x,y) using Equation (4). Both the equations of motion in physical space (1) and in modal space (7) are numerically integrated for these initial conditions using a fifth order Runge-Kutta method. The resulting time series in modal space are then transformed back to physical space and compared to the time series generated directly in physical space. The comparison is achieved using the normalized mean-square error (NMSE):

$$
\operatorname{NMSE}(\hat{f})=\frac{100}{N \sigma_{f}^{2}} \sum_{i=1}^{N}(f(i)-\hat{f}(i))^{2}
$$

where $\hat{f}$ is the time series to be compared to the reference series $f, N$ is the number of samples and $\sigma_{f}^{2}$ is the variance of the reference time series. A NMSE value of $1 \%$ is commonly assumed to reflect excellent concordance between the time series. 


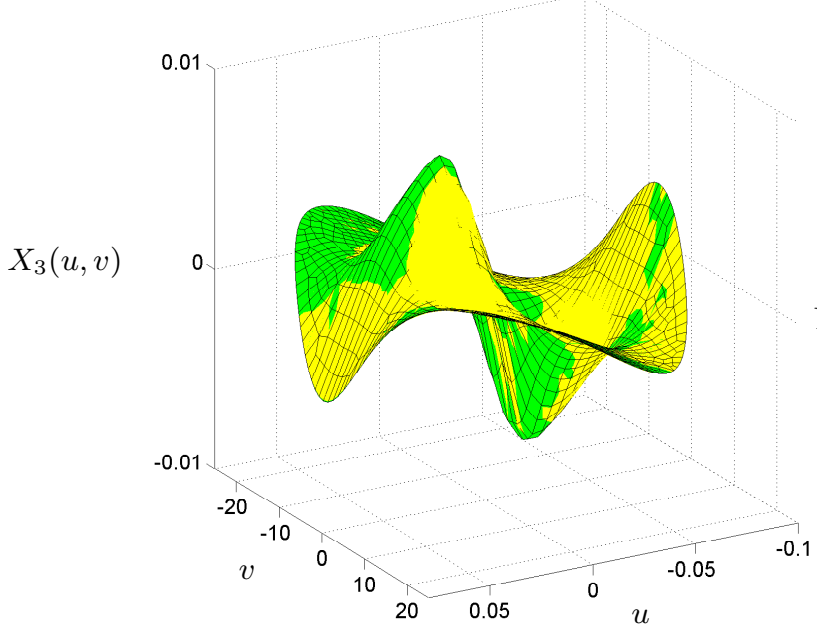

(a)

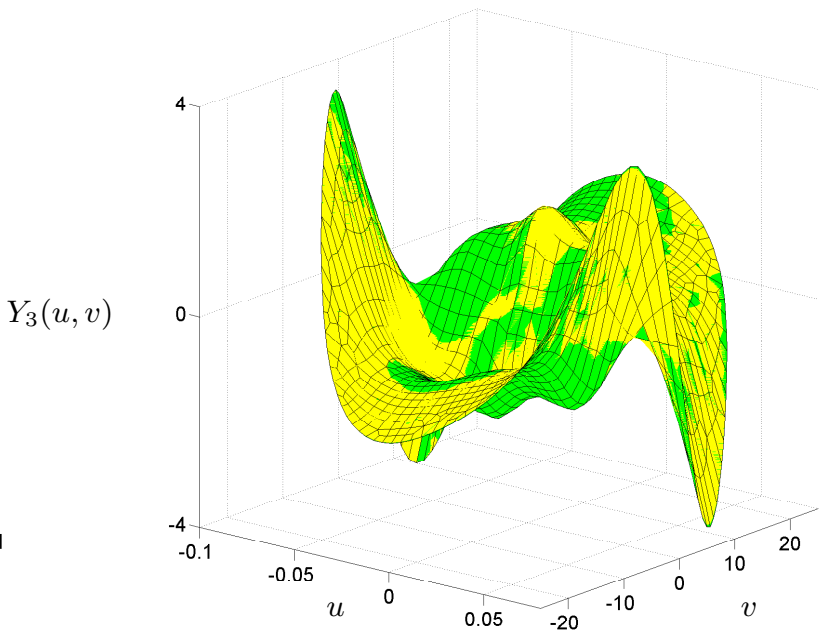

(b)

Figure 9: Second NNM for the 5DOF system. The results from the invariant manifold approach (green) are in excellent agreement with the reference (yellow). (a) Slave displacement $X_{3}$. (b) Slave velocity $Y_{3}$.

Figure 10 compares the dynamics reduced on the invariant manifold and the full-system dynamics. A excellent agreement between the different time series is observed. Despite the complexity of the time series (cf. Figure 10 (b)), the matching is conserved over more than 20 periods. It especially indicates a very accurate capture of the motion frequency (in addition to the motion amplitude capture). The NMSE of $7 \mathrm{e}-2 \%$ confirms our observations.

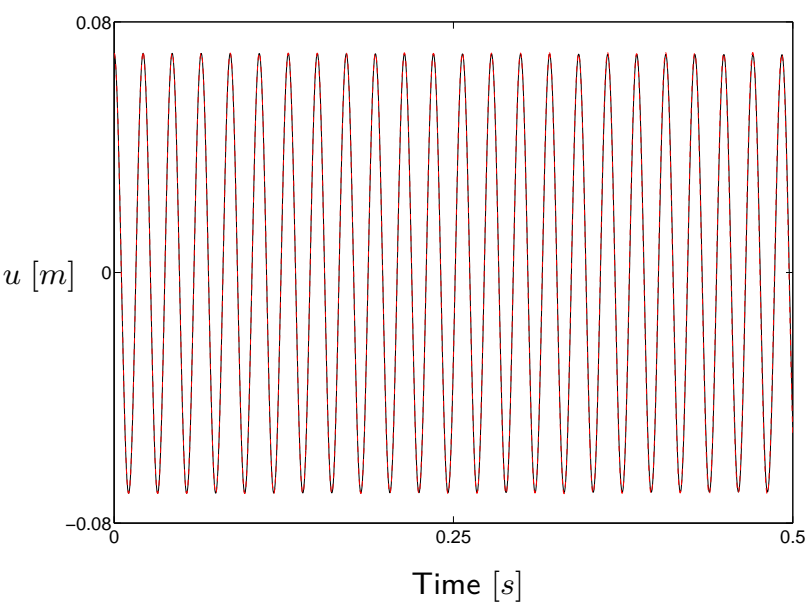

(a)

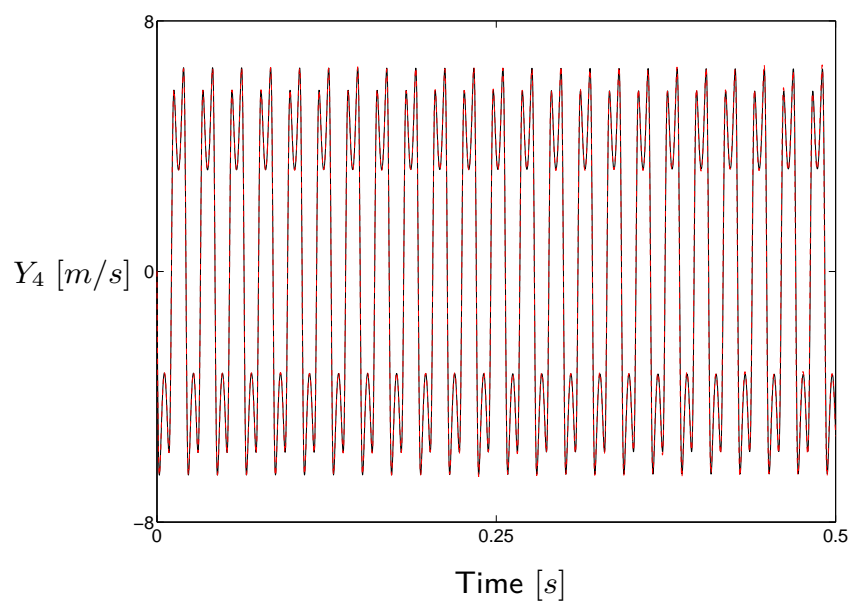

(b)

Figure 10: Comparison between the dynamics reduced on the manifold $(-)$ and the full-system dynamics $(--)$. Both time series are superimposed and the NMSE value of $7 \mathrm{e}-2 \%$ confirms the perfect matching. (a) Master displacement. (b) Slave velocity of the fourth mass. 


\section{TWO NONLINEAR NONCONSERVATIVE SYSTEMS}

In this section, we first consider a simple nonlinear system with weak linear non-proportional damping. This system is the system already presented in Figure 1 where we have introduced damping. The equations of motion are presented in Equations (21).

$$
\begin{aligned}
\ddot{x}_{1}+2 x_{1}-x_{2}+0.5 x_{1}^{3}+c_{1} \dot{x}_{1}-c_{2} \dot{x}_{2} & =0, \\
\ddot{x}_{2}+2 x_{2}-x_{1}-c_{2} \dot{x}_{1}+c_{3} \dot{x}_{2} & =0 .
\end{aligned}
$$

Figure 11 presents the second NNM of the system. This mode corresponds to an out-of-phase motion of the two masses. In the nonconservative case, reference solutions do not exist and the solution accuracy assessment is performed through the dynamics. The computation of the dynamics for various initial conditions shows NMSE values around $1 \mathrm{e}-3 \%$. This very good accuracy is illustrated in Figure 12 (a) where we observe a perfect matching between the dynamics reduced on the NNM and the full-system dynamics.

In Figure 11 (a), we observe a "hill" in the surface describing the slave displacement $X_{2}(u, v)$. This hill illustrates the localization phenomenon. Indeed, as the energy increases, both master coordinates increase whereas the slave coordinates decreases, all the energy is localized into the first mass. The localization phenomenon was already observed for the conservative system and it is now preserved in the presence of weak linear damping. The integration of the dynamics over a very long period (the system is weakly damped) and for "high-energy" initial conditions illustrates the particular behavior of $X_{2}$ (see Figure 12 (b)). Thus, despite the presence of damping, $X_{2}$ first increases and then decreases. On the surface $X_{2}(u, v)$, the time series of Figure 12 (b) corresponds to a motion that starts out close to the domain boundary and that spirals toward the origin. Each passage through the hill happens closer to the origin and first increases then decreases the motion amplitude. Exponential decay is observed for the master DOFs.

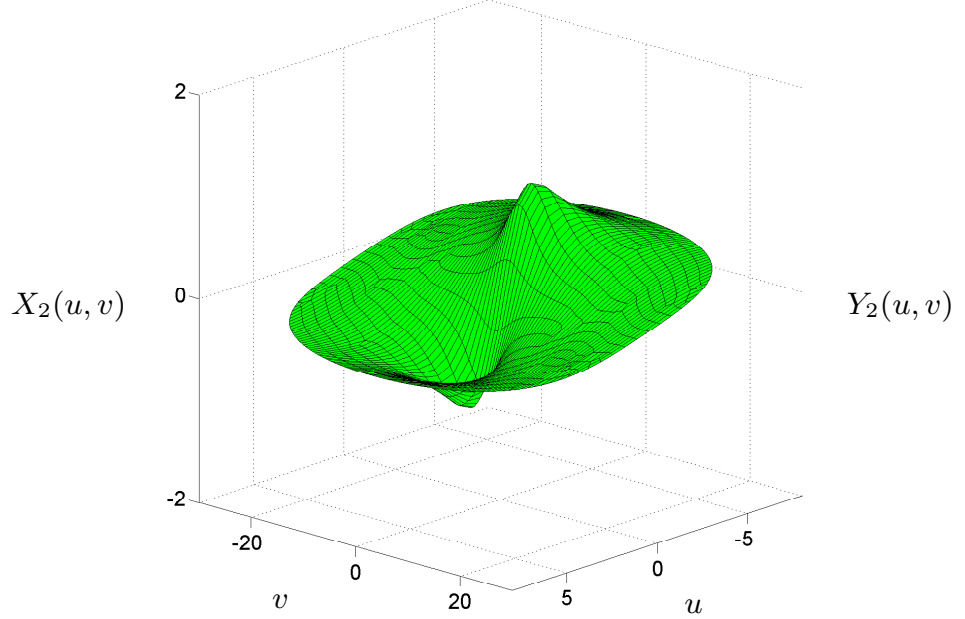

(a)

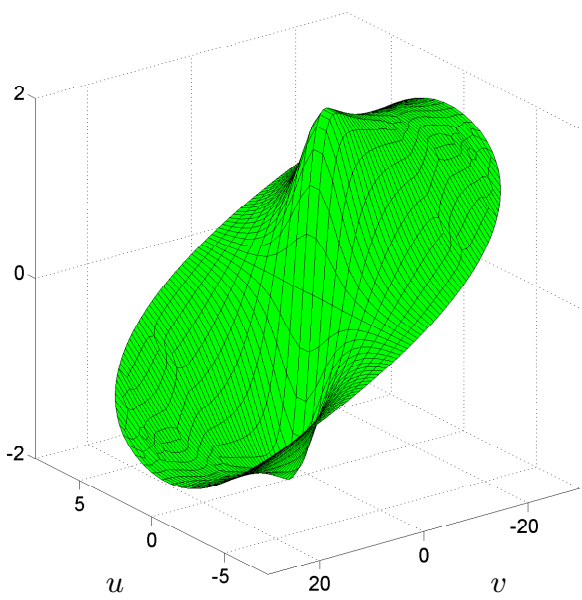

(b)

Figure 11: Second NNM for the 2DOF system with weak non-proportional linear damping. (a) Slave displacement $X_{3}$. (b) Slave velocity $Y_{3}$.

We now consider a more complicated system with 2DOF and including a cubic stiffness, negative linear damping, and cubic positive damping. The equations of motion of these coupled-Rayleigh oscillators are presented in Equations (22) where $a=0.1$ and $b=0.75$.

$$
\begin{array}{r}
\ddot{x}_{1}+2 x_{1}-x_{2}+0.5 x_{1}^{3}+b \dot{x}_{1}^{3}+b\left(\dot{x}_{1}-\dot{x}_{2}\right)^{3}-2 a \dot{x}_{1}+a \dot{x}_{2}=0 \\
\ddot{x}_{2}+2 x_{2}-x_{1}+b \dot{x}_{2}^{3}-b\left(\dot{x}_{1}-\dot{x}_{2}\right)^{3}-2 a \dot{x}_{2}+a \dot{x}_{1}=0
\end{array}
$$

Figure 13 (a) displays the invariant manifold computed from the first LNM. Results validation are performed using the dynamics. Figure 14 presents the dynamics computed for two different initial conditions. An excellent agreement is again observed and NMSE values do not exceed $3 \mathrm{e}-3 \%$. For both initial conditions, we can observe that oscillations amplitude are conserved after some transient response. The representation of time series in the $(u, v)$ plane highlights the presence of a limit cycle. We note that to properly simulate the dynamics, a fine mesh is needed around this limit cycle. 


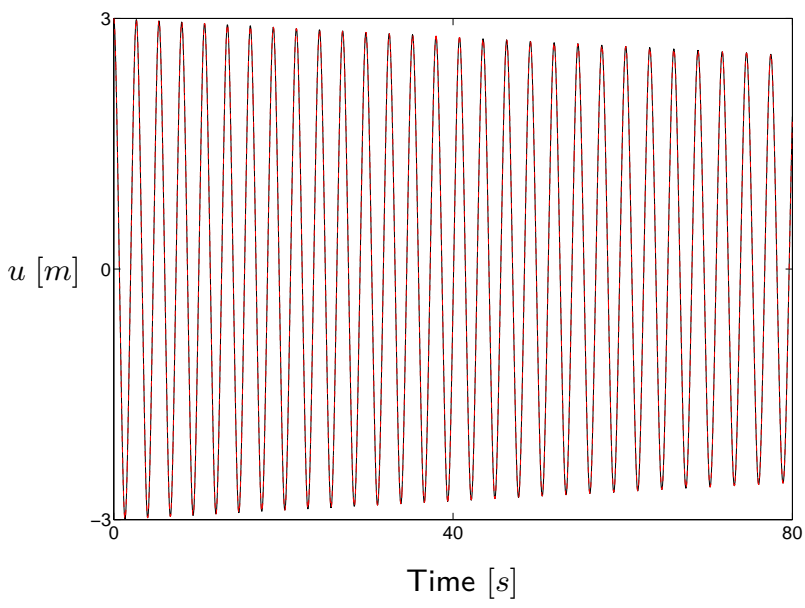

(a)

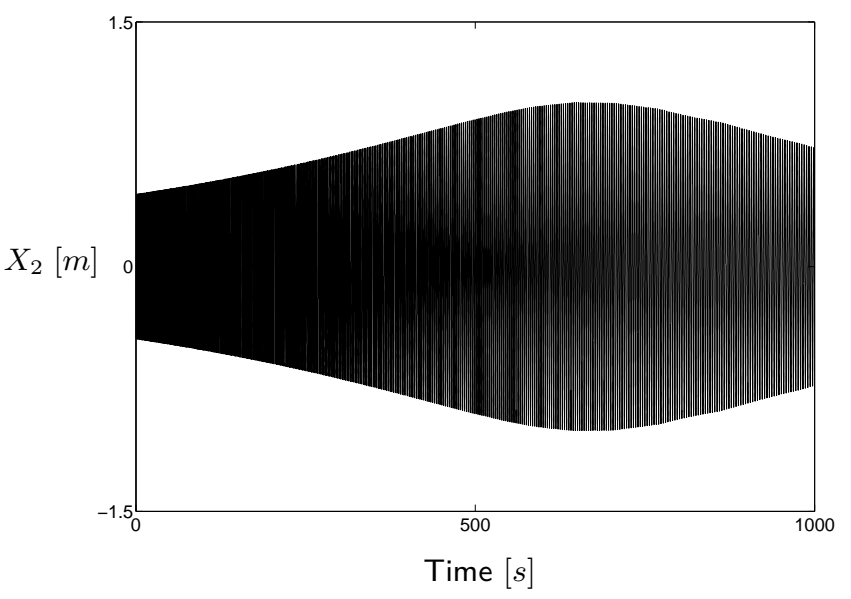

(b)

Figure 12: (a) Comparison between the dynamics reduced on the manifold $(-)$ and the full-system dynamics $(--)$. Both figures represents the master displacement $u(t)$. Initial conditions are taken as $(u, v)=(4.0,0)$. The NMSE equals 1 e-3\%. (b) Localization phenomenon observed for high-energy initial conditions $((u, v)=(6.0,0))$.

In this example, we went beyond the theoretical framework of NNMs. However, we show that the algorithm is still capable of reducing accurately the dynamics on a two-dimensional invariant manifold.

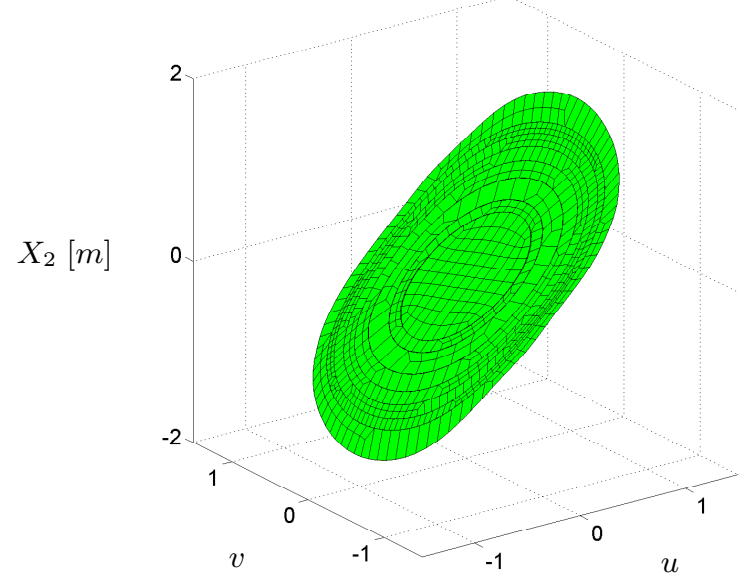

(a)

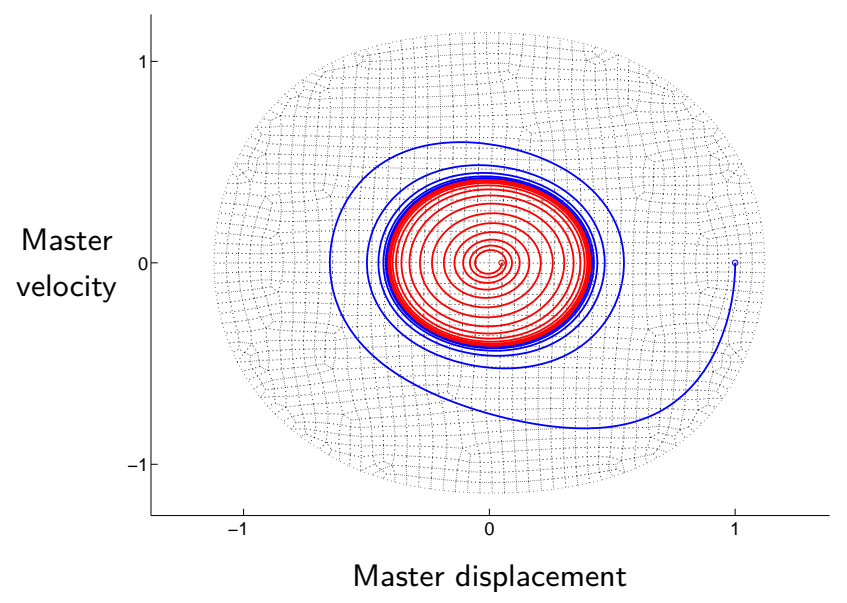

(b)

Figure 13: Invariant manifold computed from the first LNM. (a) Slave displacement of the second mass $\left(X_{2}\right)(\mathbf{b})$

Dynamics in the 2D phase plane. Two different initial conditions are considered: $(u, v)=(0.1,0)$ and $(u, v)=(0.94,0)$ and both are denoted by a circle. A limit-cycle is observed. 


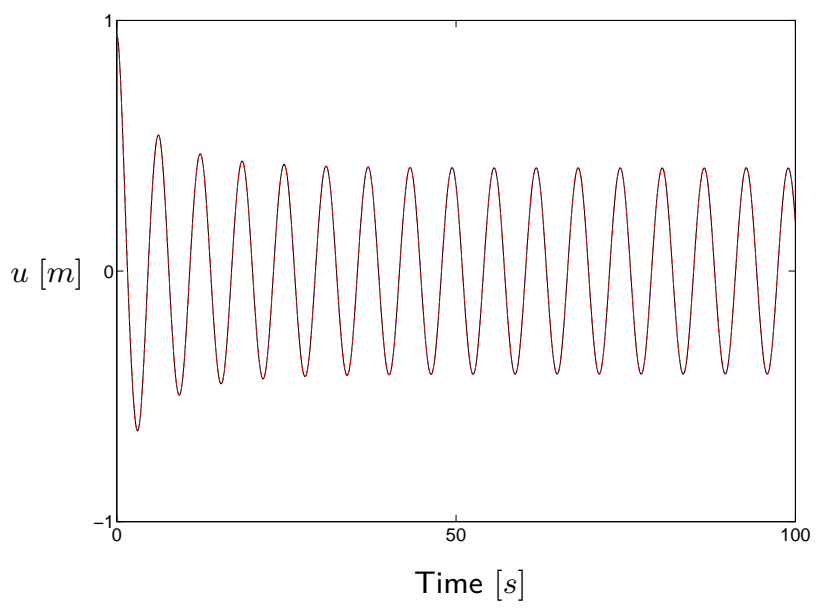

(a)

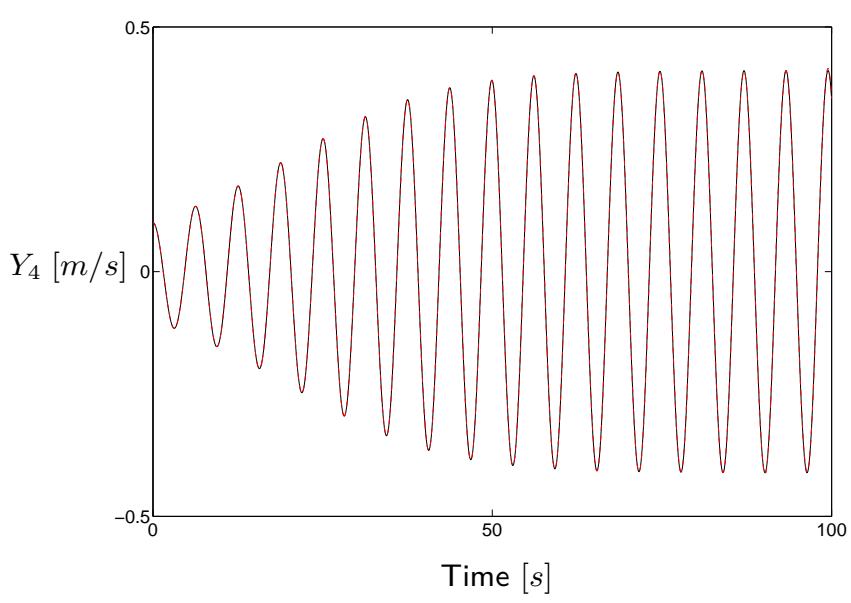

(b)

Figure 14: Comparison between the dynamics reduced on the manifold $(-)$ and the full-system dynamics $(--)$. Both figures represents the master displacement $u(t)$. (a) Initial conditions are taken as $(u, v)=(0.94,0)$. The NMSE equals 1e-3\%. (b) Initial conditions are taken as $(u, v)=(0.1,0)$. The NMSE equals $3 \mathbf{e}-\mathbf{3} \%$.

\section{CONCLUSIONS}

After a brief review of the key concepts about NNMs (Section 2), we introduced the PDEs governing the NNMs defined as an invariant manifold in phase space. Alike ${ }^{[4]}$, our formulation uses physical rather than modal coordinates. In Section 3 , we presented a novel algorithm to solve NNMs equations. We especially highlighted the hyperbolic nature of the PDEs (transport phenomenon) and, based on this observation, we proposed specific numerical treatments. The FE formulation (SUPG) is properly suited to our equations and the resolution strategy accounts for the boundary conditions using annular-ring domains with iso-energy boundaries. A mesh moving technique is employed to correct the shape of the domain. Section 4 demonstrated the algorithm on a MDOF conservative system with multiple nonlinearities. The computation of the NNM and the resulting dynamics were both very accurate. Finally, Section 5 demonstrated that the developed algorithm was applicable to nonconservative system including either linear or nonlinear damping.

Despite our algorithm can already deal with a large variety of systems, additional investigations are still needed. In particular, we note the influence of the SUPG parameter $\tau$ that is crucial to the method. Another feature of the invariant manifold approach is that the notion of time is lost and the access to the motion frequency is not straightforward. Next developments will include the computation of this frequency.

\section{ACKNOWLEDGMENTS}

The author L. Renson would like to acknowledge the Belgian National Fund for Scientific Research (FRIA fellowship) for its financial support.

\section{References}

[1] R. M. Rosenberg. On nonlinear vibrations of systems with many degrees of freedom. Advances in Applied Mechanics, Volume 9:155-242, 1966.

[2] R. M. Rosenberg. The normal modes of nonlinear n-degree-of-freedom systems. Journal of Applied Mechanics, page 8, 1962.

[3] S. W. Shaw and C. Pierre. Non-linear normal modes and invariant manifolds. Journal of Sound and Vibration, page 4, 1991.

[4] S. W. Shaw and C. Pierre. Normal modes for non-linear vibratory systems. Journal of Sound and Vibration, 164:40, 1993. 
[5] L. Jezequel and C. H. Lamarque. Analysis of non-linear dynamical systems by the normal form theory. Journal of Sound and Vibration, 149(3):429-459, 1991.

[6] M. E. King and A. F. Vakakis. An energy-based formulation for computing nonlinear normal modes in undamped continuous systems. Journal of Vibration and Acoustics, 116(3):332-340, 1994.

[7] Y.V. Mikhlin V.N. Pilipchuk A.A. Zevin A.F. Vakakis, L.I. Manevitch. Normal Modes and Localization in Nonlinear Systems. John Wiley \& Sons, New York, 1996.

[8] C. Touzé, O. Thomas, and A. Huberdeau. Asymptotic non-linear normal modes for large-amplitude vibrations of continuous structures. Computers \& Structures, 82:2671-2682, 2004.

[9] Joseph C. Slater. A numerical method for determining nonlinear normal modes. Nonlinear Dynamics, page 12, 1996.

[10] Young Sup Lee, Gaetan Kerschen, Alexander F. Vakakis, Panagiotis Panagopoulos, Lawrence Bergman, and D. Michael McFarland. Complicated dynamics of a linear oscillator with a light, essentially nonlinear attachment. Physica D: Nonlinear Phenomena, 204:41-69, 2005.

[11] Rémi Arquier, Sergio Bellizzi, Robert Bouc, and Bruno Cochelin. Two methods for the computation of nonlinear modes of vibrating systems at large amplitudes. Computers \& Structures, 84:1565-1576, 2006.

[12] Fengxia Wang and Anil Bajaj. Nonlinear normal modes in multi-mode models of an inertially coupled elastic structure. Nonlinear Dynamics, 47(1):25-47, 2007.

[13] M. Peeters, R. Viguié, G. Sérandour, G. Kerschen, and J. C. Golinval. Nonlinear normal modes, part II: Toward a practical computation using numerical continuation techniques. Mechanical Systems and Signal Processing, 23(1):195-216, 2009.

[14] M. Peeters, G. Kerschen, J. C. Golinval, and C. Stephan. Nonlinear normal modes of real-world structures: Application to a full-scale aircraft. ASME Conference Proceedings, 2011(54785):475-492, 2011.

[15] Eric Pesheck. Reduced order modeling of nonlinear structural systems using nonlinear normal modes and invariant manifolds. $\mathrm{PhD}$ thesis, 2000.

[16] E. Pesheck, C. Pierre, and S. W. Shaw. A new Galerkin-based approach for accurate non-linear normal modes through invariant manifolds. Journal of Sound and Vibration, 249(5):971-993, 2002.

[17] F. Blanc, K. Egeb, C. Touzé, J.-F. Mercier, and A.-S. Bonnet Ben Dhia. Sur le calcul numérique des modes non-linéaires, 2011.

[18] Daniel Noreland, Sergio Bellizzi, Christophe Vergez, and Robert Bouc. Nonlinear modes of clarinet-like musical instruments. Journal of Sound and Vibration, 324:983-1002, 2009.

[19] Denis Laxalde and Mathias Legrand. Nonlinear modal analysis of mechanical systems with frictionless contact interfaces. Computational Mechanics, 47(4):469-478, 2011.

[20] G. Kerschen, M. Peeters, J. C. Golinval, and A. F. Vakakis. Nonlinear normal modes, part I: A useful framework for the structural dynamicist. Mechanical Systems and Signal Processing, 23(1):170-194, 2009.

[21] Stig Larsson and Vidar Thomée. Partial Differential Equations with Numerical Methods, volume 45. Springer Berlin Heidelberg, 2003.

[22] Michael Renardy and Robert Rogers. An Introduction to Partial Differential Equations, volume 13. Springer Berlin Heidelberg, 2004.

[23] Jean Donea and Antonio Huerta. Steady Transport Problems. Finite Element Methods for Flow Problems. John Wiley \& Sons, Ltd, 2005.

[24] Alexander N. Brooks and Thomas J. R. Hughes. Streamline upwind/Petrov-Galerkin formulations for convection dominated flows with particular emphasis on the incompressible Navier-Stokes equations. Computer Methods in Applied Mechanics and Engineering, 32:199-259, 1982.

[25] T. J. R. Hughes and T. E. Tezduyar. Finite element methods for first-order hyperbolic systems with particular emphasis on the compressible Euler equations. Computer Methods in Applied Mechanics and Engineering, page 68, 1984.

[26] Petr Knobloch. On the definition of the SUPG parameter. Electronic Transactions on Numerical Analysis, 32:76-89, 2008. 
[27] Tayfun E. Tezduyar and Yasuo Osawa. Finite element stabilization parameters computed from element matrices and vectors. Computer Methods in Applied Mechanics and Engineering, 190:411-430, 2000.

[28] J. E. Akin and Tayfun E. Tezduyar. Calculation of the advective limit of the SUPG stabilization parameter for linear and higher-order elements. Computer Methods in Applied Mechanics and Engineering, 193:1909-1922, 2004.

[29] Ramon Codina, Eugenio Oñate, and Miguel Cervera. The intrinsic time for the streamline upwind/Petrov-Galerkin formulation using quadratic elements. Computer Methods in Applied Mechanics and Engineering, 94(2):239-262, 1992.

[30] Keith Stein, Tayfun E. Tezduyar, and Richard Benney. Automatic mesh update with the solid-extension mesh moving technique. Computer Methods in Applied Mechanics and Engineering, 193:2019-2032, 2004.

[31] Zhenlong $\mathrm{Xu}$ and Michael Accorsi. Finite element mesh update methods for fluid-structure interaction simulations. Finite Elements in Analysis and Design, 40:1259-1269, 2004.

[32] Bruno Cochelin and Christophe Vergez. A high order purely frequency-based harmonic balance formulation for continuation of periodic solutions. Journal of Sound and Vibration, 324:243-262, 2009. 